\title{
Retour aux modes de production, sans contrôle philosophique
}

Back to modes of production, without a philosophical control

\section{Georges Guille-Escuret}

\section{(2) OpenEdition \\ 1 Journals}

Édition électronique

URL : https://journals.openedition.org/tc/5017

DOI : 10.4000/tc.5017

ISSN : 1952-420X

Éditeur

Éditions de l'EHESS

\section{Édition imprimée}

Date de publication : 30 juin 2010

Pagination : 489-505

ISSN : 0248-6016

\section{Référence électronique}

Georges Guille-Escuret, « Retour aux modes de production, sans contrôle philosophique », Techniques \& Culture [En ligne], 54-55 | 2010, mis en ligne le 30 juin 2013, consulté le 29 septembre 2022. URL http://journals.openedition.org/tc/5017 ; DOl : https://doi.org/10.4000/tc.5017 


\section{RETOUR AUX MODES DE PRODUCTION, SANS CONTRÔLE PHILOSOPHIQUE}

in Techniques E culture 40, $2003: 81-105$

[Dans les années 1970, le marxisme a conquis - en France, au moins - une indubitable suprématie sur l'anthropologie économique et sa supériorité théorique a suscité ensuite si peu de contestations que ce sont des chapelles se réclamant du même prophète qui se sont déchirées pour accaparer la légitimité de l'inspiration et l'autorité attenante. Vingt ans après, non seulement l'anthropologie économique n'obéit plus à des maîtres marxistes, mais il n'y a pratiquement plus d'anthropologie économique: plus de communauté galvanisée par son enjeu. Des recherches dispersées, oui. Et même des tentatives d'appropriation expliquant que ce champ de recherche retrouvera vite son lustre d'antan pour peu qu'il oublie la production et se consacre aux échanges (Guille-Escuret 2001). Mais du vieil objet, du formidable programme et des magnifiques aspirations de naguère, plus rien, sinon quelques miettes piteuses.

Pourtant, la percée soudaine des problématiques marxistes dans l'économie politique des systèmes exotiques s'est révélée trop marquante pour correspondre platement au caprice d'une époque. Elles devaient avoir en elles-mêmes des ressources originales susceptibles de transformer radicalement une analyse. L'inertie du champ scientifique après l'évaporation du marxisme vient justement de la difficulté qu'éprouvent les intuitions concurrentes à prendre ou reprendre sa place: faute d'une compétence capable d'investir les lieux, on préfère s'installer à côté et, pour plus de sécurité, changer la cible. La critique de l'ethnocentrisme marginaliste, la réfutation des mécaniques fonctionnalistes guidées par des maximisations myopes, ou encore les démentis infligés à des psychologies d'intérêt assimilables à du babil théorique, tous ces coups de semonce notoirement dus au marxisme demeurent bel et bien... efficaces. En science, rien de plus facile que de lâcher 
une théorie encombrante: rayer d'un trait les conséquences du « ménage » que la théorie herculéenne avait accompli chez Augias, c'est une tout autre affaire. L'univers scientifique protège les négations mieux que les propositions et les sciences sociales n'enfreignent pas plus cette règle-là que ne le font leurs consœurs « expérimentées » (Guille-Escuret 1999).

L'ethnologie économique voudrait aujourd'hui se passer du marxisme mais il lui faut garder en mémoire la pertinence des réfutations alignées par celui-ci: elle ne parvient à harmoniser les deux aspirations qu'en renonçant à son contenu.

Au-delà de l'économie elle-même, l'écologie et la technologie sont également atteintes par la maladie. Peu importe que l'affaissement du marxisme ait facilité une répugnance généralisée à l'égard du substrat matériel de la vie sociale ou, qu'à l'inverse, cette lassitude ait asphyxié le marxisme avant l'implosion du régime soviétique: les deux aspects sont solidaires. La technologie culturelle a l'occasion de s'en rendre compte mieux que l'écologie humaine, laquelle a le loisir de se pelotonner provisoirement dans l'étude des interprétations culturelles de la nature, avec tout ce que cela implique de sémiologie. Des havres similaires existent sans doute aussi en analyse des techniques, mais ils sont plus étriqués et abriteraient difficilement toute la communauté.]

Or, l'ethnologie unifiée des fantasmes n'osait pas mépriser la technologie quand les marxistes rôdaient encore dans les conseils de rédaction et les commissions de spécialistes. Bien que formés pour la plupart en métaphysique, ceux-ci avaient trop conscience de dépendre d'une solidarité de principe avec un secteur visitant le matérialisme à ses racines. Et, par exemple, ils exprimaient une bienveillance sans faille à l'égard d'André Leroi-Gourhan, ou de son école. Avec une touche de condescendance, reconnaissons-le. Tant pis: tout indique que la protection n’a pas été inutile, depuis que les marxistes ne tapent plus du poing sur les tables. Dans certains cercles huppés où l'on aimerait faire à moindre frais de la place au cognitivisme, à la psychologie évolutionnaire, à l'amodernisme, ou à d'autres « révolutions » embryonnaires, on ne se retient plus de ranger Leroi-Gourhan parmi les vestiges paléo-ethnologiques (sans descendance théorique, s'entend). Pas en face des élèves, bien sûr, puisqu'ils sont présumés inexistants. Ces anathèmes émanent d'éminences grisâtres qui jugent et tranchent en tournant le dos aux condamnés: l'irruption d’un débat créerait un cinglant démenti.

Dans cette ambiance pénible, notre propos visera à séparer les intérêts scientifiques invariants de la problématique marxiste dans les démarches initiales de l'ethnologie économique, d'une part, des déboires récents du militantisme méta-scientifique, d'autre part; puis, à chercher ce qui pourrait raviver cette richesse. Cependant, peu importe ici que l'auteur et/ ou le lecteur soient ou non des marxistes certifiés. Refuser de singer la lutte de classes dans un amphithéâtre n'équivaut nullement à nier la dimension politique de l'activité scientifique.

\section{L'intérêt du Capital en ethnologie: un instrument sans concurrents pour la comparaison}

La présente réflexion représente moins une esquisse qu'une amorce. On en saisira mieux l'idée centrale à la lueur d'anecdotes qui ont favorisé son émergence sur des zones d'observation très éloignées l'une de l'autre, les Hautes Corbières viticoles et la forêt centrafricaine 
au sud de la Lobaye. Dans les deux cas, l'enquête comparative se fixait sur l'articulation entre facteurs environnementaux et facteurs historiques. Une série de constats s'ensuivit sur la modélisation du mode de production, d'abord en Languedoc, puis, de façon pratiquement inchangée en Afrique, cette répétition à l'identique étant à la fois déroutante et intrigante. Toutefois, avant d'exposer ces perplexités, quelques souvenirs doivent être rafraîchis. Dans leur grossièreté assumée, les remarques qui suivent profitent impudemment de l'accalmie des polémiques pour retrouver l'accès à des problèmes longtemps protégés par des armatures conceptuelles «philosophistiquées ».

Rappelons qu'au début des années 1980, plusieurs conceptions se livraient une rude concurrence. Certaines ont peu influencé les Français. D’autres n’ont pas réussi à investir notre discipline ou sont demeurés sans suite pour des motifs divers. Au bout du compte et en excluant les critiques particulières du néocolonialisme, deux tendances apparemment inconciliables ont nettement dominé la scène.

Primo, celle du clan althusserien, selon la grille d'Étienne Balibar (1968) appliquée par Emmanuel Terray (1969) aux sociétés primitives à partir de l'ouvrage déclencheur de Claude Meillassoux (1964) sur les Gouro de Côte-d'Ivoire. Ce dernier semble avoir accueilli assez favorablement la relecture. Enfin, Robert Cresswell (1975), bien que sans étiquette, a prolongé cette perspective de façon à ce que les rapports techniques soient mieux rendus dans la description. Son résumé exceptionnellement bref des principes de l'analyse du mode de production est reproduit à la fin de cet article, en annexe, pour stimuler les réminiscences (ou faciliter les... révisions).

Secundo, celle des «structuralo-marxistes »- vocable initialement péjoratif, mais peu importe -, guidés par Maurice Godelier (1973) et par Pierre Bonte (1973, 1975).

Un long récapitulatif des finesses de ces constructions ne nous aiderait pas à sortir des anciennes ornières, surtout si nous succombions à la tentation d'applaudir l'auteur le plus fidèle à la pensée fondatrice. Reconnaissons cependant que les mises au point de Terray et de Cresswell avaient sur les réflexions complexes de Godelier un avantage considérable: elles étaient directement utilisables sur le terrain. Ne serait-ce qu'en soustendant un recensement des activités économiques et en prévoyant pour chacune d'elles de mettre en valeur les relations entre technique et social. Entre autres fonctions, la formule du procès de travail donnée par Cresswell $(\mathrm{FT}+\mathrm{IP}--->\mathrm{MP}=\mathrm{P})^{1}$ préparait l'enquêteur à l'inventaire économique et complétait à merveille les tableaux élémentaires déjà réclamés en écologie humaine et en technologie (le cycle annuel des activités, par exemple).

L'intervention de la seconde tendance, en tant que moteur de discussion générale ${ }^{2}$, se plaçait donc après la collecte strictement ethnographique des données, dans les phases de comparaison et de synthèse. Avec, en point de mire, une énigme hautement privilégiée où la dissension avec la vision adverse atteignait le maximum d'intensité: la pluralité des modes de production au sein d'une formation économique-sociale. Thème fameux sur lequel on reviendra après avoir souligné certaines virtualités scientifiques du marxisme qui sont tombées dans une relative indifférence, oblitérées par l'apparent consensus qui les touchait.

Ainsi, la tripartition du mode de production, où deux formes de superstructures (idéologique et juridico-politique) sont confrontées à une instance économique, a souvent été admise d'office, puis subrepticement ramenée à une simple bifurcation entre le haut et la base, l'idéel et le matériel. La séparation entre l'idéologie et le juridico-politique prenait alors l'allure d'une coupure subalterne. Or, les idéologies parascientifiques adorent les dualités parce qu'elles peuvent les empiler à leur gré, ce qui débride les extensions analogiques et ravive les grands antagonismes binaires de la métaphysique occidentale: 
les systèmes multipolaires sont moins manipulables. Les marxistes auraient dû se méfier d'une réduction spontanée qui les reconduisait vers des schémas théoriques reniés ${ }^{3}$.

En ce qui nous concerne ici, la tripartition implique qu'il y a aussitôt deux sortes d'efficacités sociales en interaction avec l'économique, lequel comprend l'efficacité technique. Et, contrairement à ce que l'on imagine, le technologue « flaire » la nécessité de cet agencement plus promptement que le philosophe. Pour la bonne raison qu'elle émerge dans l'observation des procès de travail particuliers et non dans la conception des systèmes globaux.

Le viticulteur des Corbières qui choisit de labourer sa terre dans le sens nord-sud, après avoir épandu du désherbant dans le sens est-ouest, n'ignore pas que le pesticide est censé remplacer complètement le passage du soc et du versoir. Pourquoi agit-il ainsi? Il est techniquement poussé à accepter d'épandre le désherbant sur une partie de ses vignes: gain de temps, place grandissante prise par d'autres travaux, faiblesses du tracteur sur des parcelles escarpées... Par ailleurs, ni les rapports patrons/ouvriers ni l'entraide ne l'incitent à ménager la chèvre et le chou en combinant les deux pratiques. Curieusement, le discours justifiant cet étrange compromis fait alors appel à des besoins naturels de la terre: elle doit « respirer », par exemple, et réclame à ce titre d'être remuée. En fait, en Languedoc, dans la première moitié du xxe siècle, la conception de la viticulture tourne autour du labour: c'est l'activité légitimante et valorisante par excellence. Par opposition avec un vigneron bourguignon qui s'identifierait autant dans les tâches de la cave que dans celles de la terre. L'efficacité sociale du labour, là, s’avère idéologique et non juridico-politique (Guille-Escuret 1993).

Par contre, la création précoce des coopératives en Hautes Corbières a été décidée en fonction d'une transformation des relations locales avec le négoce du vin et malgré les compétitions acharnées entre exploitants pour l'acquisition de parcelles rentables: dans la plupart des communes, les coopératives ont été créées par des propriétaires aisés du village. Là, le technique et le juridico-politique ont stabilisé une interaction repoussant les réticences idéologiques. Dans certains cas, cependant, les aspirations idéologiques des ouvriers agricoles et les intérêts de leurs employeurs se sont opposés et ont entravé la solution technique que la coopérative donnait aux différents problèmes de stockage des uns et des autres. En ce cas précis, les situations deviennent rapidement très compliquées et variables, mais l'important, pour l'instant, est que les rapports du technique et du social sont à la fois indescriptibles et incompréhensibles si la dimension idéologique et la dimension juridico-politique sont confondues.

En forêt africaine, la concurrence de deux procès de travail a induit la même conclusion: il s'agissait de la récolte de vin de palme, traditionnellement réalisée à partir des fleurs sur l'arbre vivant ("à l'accroché ») mais récemment effectuée sur des troncs de jeunes palmiers (« au déraciné »). Pour de multiples raisons, la forme classique renforce les liens de parenté alors que la forme nouvelle menace de les corroder gravement (GuilleEscuret 1990). Chez les Ngbaka, où les tensions entre sous-lignages et entre classes d'âge sont vives, la commercialisation du vin « au déraciné » a précipité la faillite du système précolonial. Chez les Ngando, où la cohésion des relations lignagères est bien préservée, les aînés décident de permettre à un jeune en difficulté financière de déraciner quelques arbres. Et, de loin en loin, ce contraste permet de rendre compte du fait que les groupes traditionalistes des Ngando ont investi une grande somme d'énergie dans la culture annuelle et commerciale du café, alors que les Ngbaka en sont presque incapables.

Il est impossible d'énumérer en quelques lignes les paramètres à incorporer dans l'argumentation, et plus encore de les situer en relation mutuelle, mais une certitude en découle: l'image selon laquelle le social choisit entre deux techniques concurrentes, celle 
qui lui convient le mieux est non seulement simpliste mais aussi stérilisante quand, au lieu de s'attacher à un objet délimité, elle prétend à quelque généralité. Souvent, différents aspects du social se disputent un devenir à travers l'alternative technique et c'est pourquoi le technologiste est magnifiquement placé pour percevoir l'hétérogénéité des rapports dits « sociaux $»^{4}$.

Quand Robert Cresswell (1996) suggère d'analyser en détail des « verrous » composés de relations de différentes sortes qui tendent à immobiliser un dispositif technique et social, ou quand Pierre Bonte (1999) fait osciller le rite entre rapports techniques et rapports sociaux, ils s'offrent une aptitude à percevoir le social en de multiples manifestations sans décréter laquelle serait « la bonne».

Dans ces conditions, n'est-il pas souhaitable de reconsidérer la pertinence d'une théorie des superstructures à établir au-delà de frontières la démarquant à la fois de l'énoncé historique et de la matérialité sociale? Avant d'acclamer le projet, il eût fallu montrer l'aspiration des structures juridico-politiques et des structures idéologiques à une théorie unique. Et, s'il apparaît que quelques étapes intermédiaires restent à parcourir avant de penser sérieusement à engranger des contributions en vue de cette vaste synthèse, l'anthropologie aura à admettre (au sein de son instance juridico-politique propre) que la théorie espérée n'était ni tout à fait celle « des » superstructures, ni tout à fait celle « du » social.

Ceux qui ont martelé leur désaccord avec le prétendu économisme de Marx ont machiné un leurre précieux: la déformation de la problématique adverse a, en effet, servi à installer en miroir une caricature symétrique, un sociologisme broyant le fait social en une purée de relations interindividuelles... Avec évacuation des grumeaux où la matière se trouve modifiée par la culture.

La lecture quotidienne du Capital n'est pas obligatoire pour rompre avec la vision selon laquelle le social et l'économique occuperaient chacun un côté du filet: quand Michel Panoff (1985) s'intéresse au travail qui, liant le technique au social, est, selon son propre terme, « consubstantiel » à l'un et à l'autre, ou quand Jean-Luc Jamard (1993) nous recommande cette perspective « transverse » qu'il a d'ailleurs lui-même explorée aux Antilles (Giraud \& Jamard 1985), ces chercheurs ne se réfugient nullement sous l'égide du matérialisme dialectique. La tripartition du mode de production est un symptôme fort de la qualité sociologique du modèle, mais si on en reconnaît l'avantage de l'extérieur, c'est qu'il n'est pas nécessaire d'être à l'intérieur pour l'admirer.

Le monopole conquis par l'analyse marxiste se manifeste aussi sur un autre plan d'étude, bel et bien déserté celui-là : la recherche comparative à construction progressive ou à échelles variables. Reprenons l'exemple précédent: Panoff peut comparer les incidences du «travail » entre deux groupes partageant une histoire, ou entre deux moments (précolonial et colonial). Giraud et Jamard peuvent confronter Martinique et Guadeloupe en fonction de phases historiques différentes (la captation de l'une de ces îles par l'Angleterre au moment de la Révolution Française et donc, de l'abolition provisoire de l'esclavage). Mais au-delà? Sans modèle d'ensemble, la comparaison ne progresse qu'en s'appuyant prudemment sur des continuités historiques et géographiques.

Où trouvera-t-on une grille conçue pour comparer les faits sociaux avec un zoom théorique s'adaptant à la dimension de la cible choisie? Seul le structuralisme sait faire cela, mais, répétons-le, pas sur tout le social ${ }^{5}$. En cherchant des concurrences, des complémentarités, des contradictions et des contrastes à toutes les échelles spatiales et temporelles de la vie sociale, le marxisme s'est lui-même implanté en ethnologie comme mode comparatif concurrent ou complémentaire du structuralisme. Ainsi, alors que celui-ci 
ne rêve que d'invariants, l'abus latent qui, par bouffées, tente le marxisme serait plutôt celui des typologies prématurées: au lieu de dépasser l'histoire, on la fixe.

Malheureusement, dans ce continuum du contraste où le raisonnement devait voyager ouvertement du moment à l'époque, du village à la civilisation et du procès de travail à la formation économique-sociale, les étages susceptibles de convenir au mode de production s'imbibaient irrésistiblement d'une excessive importance symbolique.

\section{Docteur, je vois des dualités de modes de production partout}

Nous en arrivons à la perplexité doublement et identiquement suscitée par les terrains languedocien et africain. Dans les Corbières viticoles, la dualité des modes de production éclairée par Terray était manifestement présente. Et aussi la dualité des modes de production définie par Godelier. Cela faisait beaucoup. Malgré leur antagonisme déclaré, les deux conceptions semblaient conciliables, ce qui déjà était inattendu. L'embarras s'accrut encore après le séjour en forêt centrafricaine: en s'en tenant à la lettre, la «modalité Terray » y coexistait de nouveau avec la «modalité Godelier ». De là à croire que la dualité de modes de production est une construction assez fascinante pour obnubiler le chercheur qui a eu le malheur de se laisser séduire, il n'y a pas loin: l'idée a été sérieusement examinée, sans résultat. Restent les hypothèses suivantes:

a) Les deux modèles traitent de réalités avérées et disjointes, qui adviennent assez souvent, et l'une et l'autre, dans les sociétés humaines.

b) Les deux modèles traitent de réalités avérées et emboîtées: l'une existe dans l'autre, partiellement ou complètement, régulièrement ou sporadiquement.

c) L'un des deux schémas (au moins) est un bricolage illusoire, inconséquent, que n'importe quelle société humaine confirmera.

Les accusations portées par les principaux intéressés ne nous éclairent guère: dans le feu de la polémique, Godelier (1973 : 167) rangea la contribution de Terray au sein de l'économisme vulgaire en lui attribuant la conviction que, chez les Gouro, il y aurait « autant de modes de production que de formes de procès de travail ». Il en cite deux et leur ajoute un « etc. » aussi sibyllin que pervers. Il faudrait savoir quelle ampleur donner au terme de «formes » accolé à « procès de travail », faute de quoi la critique stagne au point mort. Celle-ci ne dépasserait la fin de non-recevoir (ou l'échange des cartes de visite) que si l'objet traité par Terray était cerné, en précisant le cas échéant pourquoi il en a mal parlé ou pourquoi l'objectif est dérisoire.

Plus instructifs sont les ensembles sociaux qui illustrent les analyses. Les Gouro sont regardés par Terray au niveau de communautés villageoises et d'activités chroniques, c'est-à-dire dans des cadres de référence restreints - infra-historiques, pour ainsi dire, puisque c'est le fonctionnement normal dans une structure qui nous est donné à voir. Pour l'Irlande, Cresswell s'appuie sur plusieurs cadres également réduits, mais en des époques différentes. En revanche, les cas évoqués par Godelier sont la France du xix siècle (maintien de rapports «précapitalistes » à côté du mode dominant), ou l'Empire inca (mode de production asiatique): l'échelle a changé. 
Il semble que l'on puisse s'avancer vers le mode de production par le bas ou par le haut. Par le bas, sous l'histoire, en progressant des procès de travail vers le modèle synthétique. La dialectique des efficacités techniques et des efficacités sociales dévoile là ses déterminations intrinsèques, les verrous qu'elle forge, les évolutions qu'elle engendre. Par le haut, en démontant la formation sociale en son histoire jusqu'à isoler son moteur et les modifications qu'il a subies. À l'horizon, une image du social se profilerait dans une dialectique entre les contraintes matérielles (écologiques ou techniques) et les événements historiques.

Les deux pistes valent d'être suivies, sans doute. Afin, au moins, de vérifier qu'elles ne se rejoignent pas à mi-chemin et qu'elles sont différentes de bout en bout: l'issue contraire ferait gagner aux sciences sociales une méthode éprouvée parcourant dans les deux sens la distance entre l'élémentaire et le global.

Passons aux enseignements annoncés des enquêtes en Languedoc et en Afrique centrale.

\section{Viticulteurs et vignerons, exploitants et coopérateurs}

Tout au long du xix siècle, la viticulture qui s'installe en Bas Languedoc fait table rase des traditions vigneronnes de la région pour imposer le système agronomique du Nord à la faveur de soudaines crises détruisant le vignoble (oïdium, phylloxera...) : aux yeux de l'ingénieur, l'optimum fixe un cépage pour chaque terre et un cru se discerne d'une année sur l'autre pour chaque lieu. La compétence agricole du viticulteur est seulement « corrigée», mais les savoirs vinicoles des vignerons languedociens sont bafoués. Peu à peu, l'élevage du vin revient aux talents du négociant, lequel crée « ses » vins par mélange des récoltes achetées.

Énumérons les types d'exploitation viticole dans les Corbières viticoles du $\mathrm{xx}^{\mathrm{e}}$ siècle en distinguant a priori deux productions successives: celle du raisin (viticole) et celle du vin (vinicole).

a) Château ou campagne: le raisin est produit sur un domaine d'un seul tenant, ou presque; le vin est produit en cave particulière. En deçà du propriétaire et/ou de son régisseur, le responsable technique des vignes et le responsable technique des chais ne sont pas la même personne. Après la Seconde Guerre mondiale, le château reconquiert tout le pouvoir sur la fabrication du vin, l'étape ultime étant la « mise en bouteilles sur la propriété ». Les « campagnes » sont autour des villages et rivalisent avec eux: jadis, on y comptait le nombre d'enfants qu'il y manquait pour avoir le droit à une école.

b) Exploitation villageoise 1: raisin sur parcelles dispersées sur le territoire de la commune (voire sur deux ou trois communes); vin complètement élaboré en cave particulière. En général, ce sont les familles les plus riches de l'agglomération, avec mise en danger de la propriété à chaque génération au moment du partage. Outre le propriétaire et, éventuellement, un ou deux fils, la main-d'œuvre (nombreuse avant la mécanisation) est bien entendu salariée. Là aussi, un seuil important est la mise en bouteilles sur la propriété grâce à quoi celle-ci acquiert une identité durable.

c) Exploitation villageoise 2: raisin sur parcelles dispersées; vin partiellement élaboré en cave particulière, avec relais du négoce. Taille de l'exploitation variable mais toujours assez grande pour mobiliser entièrement le travail d'au moins un homme.

d) Exploitation villageoise 3: raisin sur parcelles dispersées; vin complètement élaboré 
en cave coopérative. Taille de l'exploitation variable. Éventuellement insuffisante pour la capacité de travail d'un homme. Il peut s'agir d'un commerçant du village, ou d'un ouvrier agricole qui exploite quelques parcelles à temps partiel.

e) Exploitation villageoise 4: raisin sur parcelles dispersées; vin partiellement élaboré en cave coopérative, avec relais du négoce. La coopérative vend son vin par cuves et est donc entièrement tributaire des humeurs du négoce.

f) Exploitation villageoise 5: raisin sur parcelles dispersées; vin partiellement élaboré dans une cave particulière n'appartenant pas au viticulteur, avec relais du négoce. C'est un cas qui tombe en désuétude avec la généralisation des caves coopératives: celui des ouvriers dont le patron prenait la récolte dans ses cuves (le mélange avec sa propre vendange étant souvent, qualitativement, à son avantage).

Entrevoit-on les symptômes d'une dualité de modes de production au sens établi par Godelier? Oui : la persistance de rapports de production précapitalistes (l'entraide), en dépit de la domination croissante du capitalisme, est flagrante ${ }^{6}$. Certaines agglomérations ont une cohésion durablement garantie par la lutte contre un château sur leur territoire, avec la récupération d'une grande surface en « landes » susceptible d'être ouverte aux troupeaux ou défrichée pour des plantations. D'autres communes vivent dans une lutte de classes intense et permanente qui laisse des traces longtemps après que la mécanisation des labours a fait diminuer la population salariée, une partie du village étant issue d'ouvriers qui achetèrent peu à peu des parcelles sur les coteaux (et qui, ultérieurement, obtinrent en priorité les labels d'appellation contrôlée). Autre style encore: un chef-lieu de canton, contenant une proportion importante de petites exploitations auxquelles s'ajoutent les embryons de propriétés qui appartiennent à plusieurs dizaines de commerçants divers se trouve disposer d'une telle masse humaine entre ouvriers et patrons que, non seulement l'entraide y est pratiquement plus facile techniquement et politiquement, mais aussi elle est devenue une valeur emblématique du bourg contre ses voisins et ennemis séculaires, passionnément hiérarchiques. Or, cette tendance à la coopération a permis aux caves coopératives qu'elle imprégnait de s'adapter au marché beaucoup plus efficacement que leurs homologues des villages hantés par leurs inégalités: en quelque sorte, l'entraide fait du coopérateur l'actionnaire modèle d'une entreprise hardie.

Du côté de la grille Balibar-Terray, une «manière de produire » viticole s'oppose techniquement et socialement à une manière vinicole. Le terroir est en proie à des compétitions interindividuelles et interfamiliales pour l'acquisition des propriétés cultivables: mariages, partages (ou héritages), ventes, achats, remembrements déterminent le comportement de celui qui fait du raisin. Cependant, quand il s'agit de faire du vin, la solidarité de tout le village dans la coopérative est indispensable à une bonne défense contre les mauvais tours des négociants. Deux logiques de production, techniquement consécutives mais socialement contradictoires, s'imbriquent dans la vie de la collectivité rurale.

Et, si cela ne suffisait pas, une troisième divergence combine traits techniques et traits sociaux. Dans un château, il n'y a qu'une seule production: les décisions agronomiques concernant les souches et les décisions œnologiques quant aux moûts incombent finalement à une seule entité responsable. Entre autres choses, cela facilite l'installation de stratégies à long terme. Au village, il y a deux productions: celle du 
raisin revient à un exploitant, celle du vin est effectuée par un ingénieur au service de l'assemblée générale. Quand on veut signifier qu'une commune ne parvient pas à résoudre ses tensions internes, le même constat revient: là-bas, « ils ont eu dix présidents de coopérative en dix ans ». On devine les effets d'une telle fébrilité sur un plan d'amélioration de l'encépagement. Nous reviendrons bientôt sur cette question dans un cadre plus adéquat.

\section{Horticulteurs villageois, Pygmées cueilleurs et Pygmées cultivateurs}

Au sud de la rivière Lobaye, en forêt centrafricaine, une série de formations sociales traditionnelles sont composées de collectivités rurales (villageois sédentaires ou, avant la colonisation, semi-nomades) et de groupes aka, chasseurs-cueilleurs pygmées vivant en campements sur les pistes de ceux qui se considèrent comme leurs « maîtres ». Passons sur la complexité de ces relations d'autorité et d'interdépendances socio-écologiques: nous sommes sans équivoque dans une situation de dualité de modes de production, au sens reconnu par Godelier. Pluralité qui s'accroît d'une unité avec l'entrée en lice du mode de production colonial.

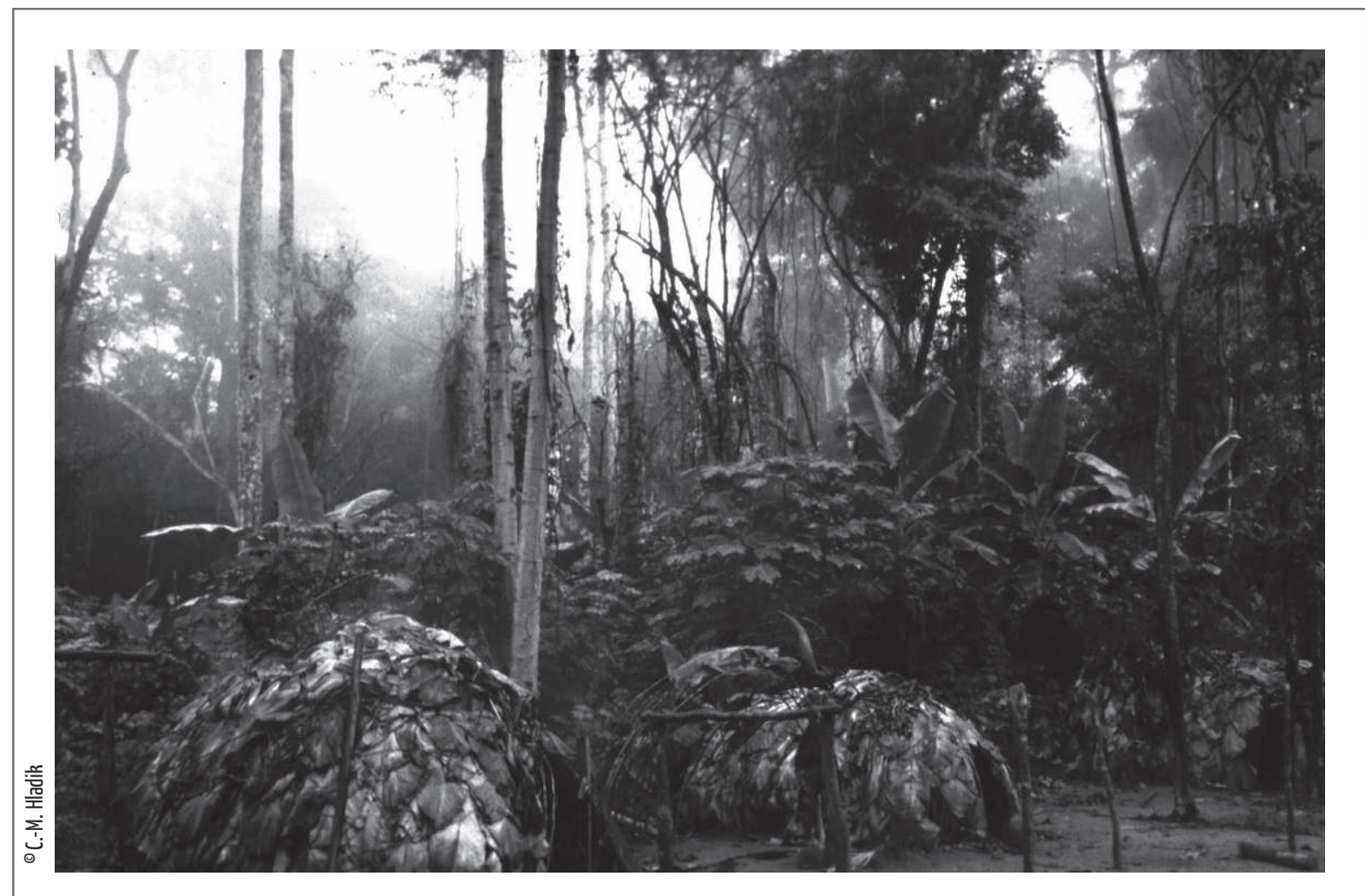

Concentrons-nous maintenant sur les villageois (en ce qui nous concerne, des Ngbaka et des Ngando): comme chez les Gouro, mutatis mutandis, la distinction se révèle fortement entre, d'une part, des activités régentées par le lignage ou le sous-lignage (l'agriculture 
vivrière, les camps de chasse pendant la saison des chenilles) et, d'autre part, des coopérations complexes qui impliquent - à l'image de la guerre d'antan - la communauté locale dans son ensemble. Nous avons donc une dualité de modes de production homologue à celle dont parle Terray dans son texte.

\section{De la dualité des dualités à la multiplicité des pluralités}

Aucun sortilège ni hasard n'explique que les deux dualités s'avèrent présentes sur l'un et l'autre terrain. L'improbable, serait qu'il en aille autrement dans un troisième lieu d'enquête. La « dualité Terray » et les «dualités Godelier » partagent un trait: elles sont également courantes et il est vraiment étonnant que cela ait si peu retenu l'attention. Y a-t-il beaucoup de régions où des relations sociales fondées sur le voisinage spatial ne composent pas avec des relations articulées par la parenté? Y en a-t-il beaucoup où la société ne se protège pas en faisant jouer simultanément ces deux registres et en combinant divers modes de coopération? Cela pour la version Terray, mais on dira la même chose de la version Godelier en transposant le raisonnement sur un axe diachronique.

Les marxistes ont tellement poursuivi le «mode de production»-vrai, immaculé, authentifié - qu'ils ont mésestimé le fait déconcertant de l'entrée du modèle en ethnologie: la thèse rejetée parlait autant que la thèse adoptée d'une réalité concrète. Il s'agissait non pas de deux thèses dont l'une serait vraie et l'autre fausse, mais de deux thèses vraies nommant pareillement deux objets différents. Éventuellement, l'une des propositions théoriques se trompait de cible, parce qu'à son insu, elle en avait découvert une. Au pire, les deux visions étaient biaisées par l'existence inconnue de deux objectifs.

Ironiquement, Balibar et Terray privilégient une approche logique et structurale, alors que Godelier, disciple de Lévi-Strauss, conçoit la dualité sous l'angle historique (substitution, domination, captation...), annonçant ainsi l'intérêt qu'il portera ensuite à la problématique de la «transition ». Entre logique et histoire, les communications sont notoirement malaisées mais les technologistes se montrent moins enclins à se résigner devant cette malédiction (Jamard 1993, Cresswell 1996).

Poussons le bouchon plus loin. Traditionnellement, la morphologie sociale des Ngbaka et des Ngando présente une alternance saisonnière: dispersion dans la forêt en petits campements de chasse sub-lignagers au moment de la saison de récolte des chenilles, regroupement au village le reste du temps. Le registre de coopération change entre les phases, et la nature technico-sociale du contraste entre les camps d'été et la vie "hivernale » se transforme profondément tout au long du xxe siècle: la permanence de la guerre aux temps précoloniaux, ou l'extension d'une culture commerciale annuelle telle que le café dans la seconde moitié du xxe siècle, modifient l'ensemble des relations. À l'instar des Esquimaux sous la loupe de Mauss, ces groupes changent donc d'état d'esprit et d'organisation matérielle au cours de l'année. Peu importe alors que l'idée d'une continuité entre mode de vie et mode de production ne bénéficie d'aucune crédibilité; si quelqu'un souhaitait soutenir l'idée d'un « mode de production saisonnier », le modèle de Balibar ne fournirait pas de quoi interdire cette interprétation et le démenti devrait recourir à l'intuition. Tout porte à croire que des rouages manquent sur les épures. 


\section{L'escamotage des procès de production et la dualité concep- tuelle des modes de production}

Du moins, y a-t-il un niveau inscrit sur le plan sur lequel les théoriciens ne se sont pas arrêtés: entre procès de travail et mode de production, le concept de procès de production est aussi important et déterminant que le deuxième étage dans une maison qui en comporte trois. Terray (1969 : 98) écrivait:

« Forces productives et rapports de production sont deux formes conjointes d'un seul et même procès mettant en jeu les mêmes facteurs; et c'est la manière spécifique dont est assurée, dans le procès de production, l'unité de ces deux formes qui fonde et détermine l'identité du mode de production. »

Bon, mais l'identité de ce mode de production ne peut-elle pas reposer sur une combinaison de procès de production? Bien sûr que si. Ou alors, toute la problématique cesserait d'avoir une quelconque utilité en sociologie comparative. Si l'inventaire et la description minutieuse des procès de travail ont un sens, on voit mal que ces tâches débouchent aussitôt sur un concept qui, dans la France du $\mathrm{xIX}^{\mathrm{e}}$ ou du xx siècle, s’appliquera pareillement à une zone de polyculture et à une aire de monoculture.

Une hypothèse s'ensuit: Terray aurait montré chez les Gouro une dualité de procès de production, constitutive d'un mode de production, tandis que Godelier aurait effectivement parlé, en France ou dans l'Empire inca, d'une dualité de modes de production. D'autant que, pour Marx, nous dit Balibar (1968 : 112), tous les modes de production sont des « moments historiques » et l'historicité est certes omniprésente dans les argumentations de Godelier.

L'ennui, c'est que cette dimension n'émerge justement pas dans la reconstitution du système gouro par Terray. Ne nous leurrons pas. Ce mode de production, désormais conçu comme combinaison de deux types de procès de production, demeure différent de la modalité historique à laquelle Godelier et d'autres se sont référés. Mode formel de production et mode historique de production sont les pôles d'un concept étendu dont nous avons déjà aperçu la tension interne: logique et histoire, atomisation et processus, structure et transformation. Des effets de manche ont fait croire que le marxisme avait dépassé ce faisceau de difficultés, alors que l'un de ses grands mérites consista à faire de la distance entre processus et structure un objet de conscience.

Libérés de la fascination exercée par le mode de production comme support de légitimation dans le marxisme et truchement pour son appropriation, les chercheurs devront prendre en compte le fait que, dans la succession des niveaux d'analyse (procès de travail, procès de production, mode formel et mode historique de production), tous sont primordiaux. Chacun d'eux domine une perspective et chacun d'eux impose sa puissance face à des interrogations spécifiques.

En revanche, il y a maintenant un retardataire à privilégier au nom de l'urgence: le procès de production, palier des collaborations interdisciplinaires. Il installe une courroie de transmission entre milieux et histoire. Et une instance de négociation entre divers aspects des rapports sociaux.

\section{L’art de tirer avantage d'un mode de production antérieur}

Revenons aux Corbières viticoles. Une pluralité «typiquement régionale » des modes de production (avec, en plus, des variations d'un village à l'autre dans le choix du 
«dominant ») serait absurde. À quoi ressemblerait la formation sociale-économique résultante? Replaçons alors la disparité à la hauteur des procès de production: si l'opération n'est qu'un ajustement illusoire, l'absurdité persistera.

La caractéristique du « château », ou de la "campagne ", c'est d'avoir sauvé l'unité du métier de vigneron. Il n'y a qu'un seul procès de production où travail viticole et travail vinicole se succèdent sans rupture, ni politique ni idéologique, sinon sans rupture technique. Au village, il y a deux procès de production - viticole et vinicole -, qui sont toujours socialement contradictoires: compétition familiale sur la terre contre solidarité villageoise à la coopérative; ou encore, le fruit du travail salarié de l'ouvrier qui rejoint son travail «à temps perdu » dans la cave particulière de son patron.

En détaillant les relations entre facteurs techniques et facteurs sociaux, on voit alors émerger leurs altérations et l'on ouvre le raisonnement sur une historicité qui s'annonce bien, cette fois, comme celle du mode de production. Mieux, il devient visible que les succès sporadiques de la viticulture des Corbières découlent de l'utilisation d'un mode de production contre un autre.

Il faut réaliser, en effet, que le mode de production capitaliste est entièrement responsable de la cassure entre le viticole et le vinicole en Languedoc. Là où une compétence vigneronne existait avant la Révolution, elle s'étendait sur les deux activités. L'évolution vers la monoculture s'accompagne d'un accaparement du vinicole par le négoce, notamment en raison de la capacité de stockage qui manque aux paysans. Les châteaux échappent à cette emprise et gardent le contrôle sur « leur » vin grâce à une situation héritée de l'Ancien Régime, et préservée.

Quant aux villages, ceux où le travail sur les parcelles s'engage le plus dans les règles de l'agriculture capitaliste débouchent sur le destin le moins enviable dans la seconde moitié du $\mathrm{xx}^{e}$ siècle, car aucune décision prise dans la coopérative n'y est à l'abri d'un revirement au cours des mois suivants. Les antagonismes sociaux vont donc à l'encontre d'une exigence technique de l'entreprise vinicole: la nécessité de maintenir des stratégies sur le long terme. Inversement, les villages qui ont su mobiliser l'entraide comme valeur éminente - principe moral apte à réduire des compétitions entre propriétaires - parviennent à reconquérir collectivement la responsabilité politique sur le procès vinicole, puis à assurer une plus-value substantielle pour leurs appellations contrôlées. La bonne gestion du procès vinicole s'avère, sur les coteaux aux maigres rendements, plus profitable qu'un productivisme viticole propice à des crises de mévente qui le rendent dérisoire.

On voit que l'observation ainsi promise du procès de production permet d'enregistrer conjointement l'action de paramètres écologiques et historiques qui semblaient jusque-là lointains et inconciliables.

\section{L'inventaire ethnographique des procès de production}

L'exemple précédent a indiqué des articulations centrales dans un cadre spécialisé: la monoculture. Un écosystème généralisé tel que la forêt centrafricaine fournit l'illustration complémentaire par excellence. Nous avons vu qu'en Lobaye, les modes de production répartissent leurs dualités sur deux axes: horticulteurs face à collecteurs, d'une part, et agriculture de subsistance face à agriculture commerciale, d'autre part. La complexité s'est encore accrue d'un degré quand, à partir du début des années 1970, les Pygmées aka « appartenant » aux Ngbaka ou aux Ngando ont multiplié les essais d'agriculture, une 
majorité de ces expériences ayant abouti à une conversion durable, avec des dispositions techno-économiques diverses (Guille-Escuret 1998). [...]

La figure 1 résume l'ensemble. Par commodité provisoire et par souci de lisibilité, la chasse y a été inscrite dans un seul cercle aussi bien chez les villageois que chez les Pygmées, alors qu'en fait elle correspond à plusieurs procès de production. Et les types de relations entre activités ont été réduits à trois: contradiction, solidarité, tension de nature variable. Le schéma montre que les deux activités associées à des transformations

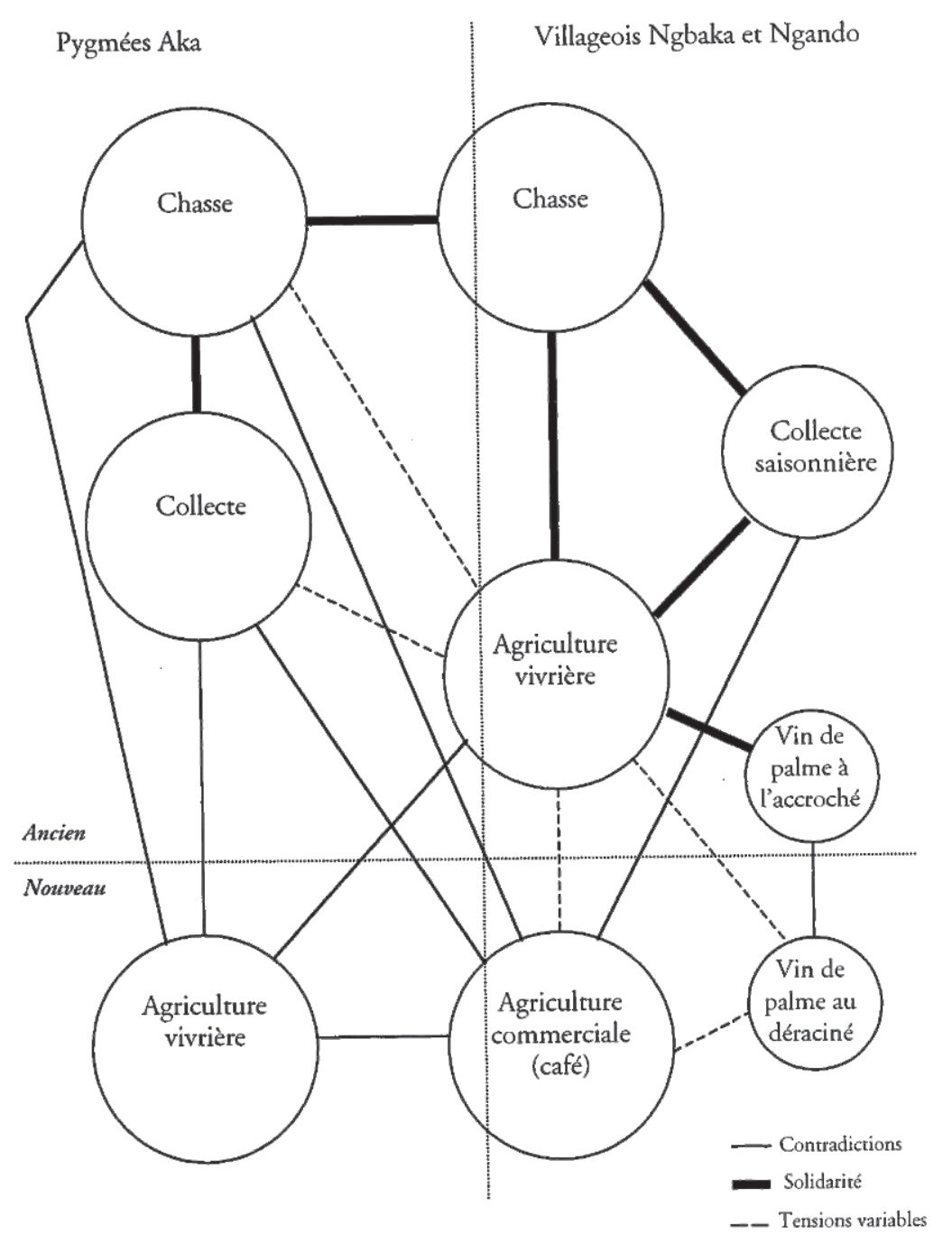

\section{Relations entre activités économiques en forêt africaine, au sud de la Lobaye}

À gauche, les activités de Pygmées; à droite, celles des villageois.

Les cercles qui empiètent sur la gauche indiquent le recours à la force de travail des Pygmées.

En haut, les activités « traditionnelles » maintenues; en bas, les activités récemment assimilées. (Fig. 1) 
historiques identifiables en termes de mode de production - agriculture vivrière pygmée (sous-entendu, loin des villages) - et plantations de café entretiennent des relations d'opposition avec toutes les tâches traditionnelles. Et elles s'embarrassent mutuellement au point que les jeunes planteurs ngando songent à enseigner les vertus de la monnaie aux Pygmées en vue de les « attacher » au café grâce à une rémunération, quitte à tolérer leurs cultures vivrières à proximité du village, la chasse étant alors à son tour fragilisée.

Ce schéma n'est pas censé montrer comment procéderait la méthodologie marxiste, car le concours de celle-ci n'y est nullement indispensable: le fonctionnalisme ou l'économisme auraient suffi. Mais justement, hors des intuitions pénétrantes d'un empirisme blanchi sous le harnais, le fonctionnalisme et l'économisme n'iront pas plus loin. L'analyse ne peut s'affiner qu'en « redescendant » vers les procès de travail simples ou complexes qui sont impliqués dans ces cercles. Il faut en dresser la liste, recenser leurs contradictions et leurs solidarités, les regrouper ou les dissocier. Dans une parcelle destinée aux biens de subsistance en Lobaye, techniquement, l'igname et le taro se confondent presque: cela ne change pas sensiblement le travail que de faire pousser l'une de ces ressources, ou bien les deux. En est-il de même pour le manioc, plante dont les tubercules sont toxiques s'ils ne sont pas traités et dont on mange les feuilles? Et puis il y a le bananier, tout à fait différent et pourtant si lié... L’ananas, le maïs, etc.

Combien de procès de travail, de produits, et de procès de production? Y a-t-il des convergences entre les organisations d'activités pratiquement lointaines (comme, chez les Gouro, la chasse au filet et la guerre)? L'ethnologie économique doit en somme réapprendre à faire ses gammes: non seulement à définir un procès de production dans ses rapports techniques et sociaux, mais aussi à attribuer un procès de production à un certain nombre de produits terminaux, distinguer ses variantes potentielles, ses concurrents, etc. Afin de planifier la comparaison à la manière des biologistes: ce n'est assurément pas simple.

\section{Conclusion d'une amorce sans amorce de conclusion}

Résumons en évitant d'adopter encore des formules ésotériques, quitte à admettre une lourdeur accrue de l'expression: le fonctionnalisme suffit à dessiner la figure 1, mais celle-ci n’a de sens que pour préparer un déplacement de perspective qui fera passer d'une typologie figée des activités à une typologie adaptable des réseaux de rapports techniques et sociaux dans la production. Adaptable sur l'éventail des échelles spatiales et temporelles du social, s'entend.

La définition d'un mode de production (puis d'une formation sociale-économique) est un aboutissement éventuel de l'examen des procès de production et non une finalité inéluctable. Du moins pour une ethnologie décidée à demeurer une ethnologie: il en va autrement pour un marxisme visitant l'ethnologie à ses propres fins: un chercheur est libre d'osciller entre ces orientations, et d'abandonner périodiquement l'une en faveur de l'autre (pour peu qu'il le fasse savoir), mais une argumentation et un programme de recherche exigent un choix déclaré en la matière. La simultanéité ruinerait l'effort. Si, au niveau du mode de production, la fresque historique peut nourrir des thèses politiquement révolutionnaires, à l'étage du procès de production, l'étude est amenée à aborder le registre de la réforme, avec son occupant permanent: la recherche appliquée. 
Plus concrètement, ce qu'une grille d'analyse privilégiant la reconnaissance des procès de production ajoutera à la figure 1 , ce n'est pas une multiplication des cercles qui désignent les activités (bien que leur nombre soit effectivement insuffisant), mais un meilleur discernement des rapports entre ces ensembles. Ainsi, contrairement à ce que l'on pourrait croire, la contradiction entre les deux agricultures vivrières - villageoise et pygmée - n'est pas techno-économique : la générosité du milieu aidant, les Aka réalisent leurs travaux avec un mois d'avance sur ceux qu'ils effectuent pour le compte de leurs « maîtres ». En fait, l'agitation atteint l'instance juridico-politique quand l'autonomie croissante des Pygmées attise l'animosité et la crainte d'un effritement de l'autorité. En revanche, entre agriculture pygmée et plantations de café, les achoppements sont bien plus complexes.

- Si les Aka défrichent près du village, ils se trouvent régulièrement à proximité des parcelles de café, mais, comme on l'a dit, la chasse en pâtit; d'un autre côté, une contradiction émergera entre l'agriculture vivrière des villageois et les plantations commerciales, puisqu'aux rapports traditionnels impliqués par la première s'opposeront les relations salariales que les secondes tendent à développer.

- Quand les Aka défrichent au loin, les Ngbaka réagissent avec d'autant plus de violence que leur cohésion lignagère est fortement meurtrie par d'incessants conflits internes; les Ngando, plus sereins dans les rapports entre leurs groupes de parenté, se montrent aussi plus diplomates avec leurs « serviteurs». Il n'est d'ailleurs pas sans intérêt de se procurer des plantes cultivées par les Pygmées sur les lieux des campements d'été (i.e. la période des chenilles, évoquée plus haut). C'est alors entre cette activité saisonnière et le café que la tension s'accroît. On voit là qu’un simple va-et-vient entre la base économique et la superstructure idéelle étoufferait dans l'œuf toute compréhension de la situation. La figure 1 rassemble l'optimum d'information sous forme visuelle mais elle est très loin de suffire: une méthodologie moins succincte doit prendre le relais pour que les sciences concernées par l'homme se rencontrent d'égales à égales, sur des relations établies dans des contextes délimités.

Il est permis de supposer que le niveau des procès de production y obtiendra la place qui l'attendait, celle de synthèses intermédiaires. Un registre où l'écologie humaine, les sciences de la cognition, les recherches sur l'innovation et les transferts de technologie, sans oublier les chantiers de recherche appliquée, ne seront plus obligés de décider à l'avance l'instance du mode de production qu'il conviendra de regarder en priorité. On sortira de l'économisme vulgaire (au nom de l'efficacité sociale) en se libérant conjointement d'un culturalisme précieux mais on se sauvera de celui-ci (au nom de l'efficacité technique) en ne replongeant pas dans celui-là. 
1. FT est la force de travail; IP signifie instrument de production; MP, matière première; et $\mathrm{P}$, produit. Dans un procès de travail complexe (agricole, par exemple) se décomposant en une série de $n$ procès simples, la formule doit être répétée $n$ fois, en considérant le résultat de chaque « travail » comme un produit $(\mathrm{P} 1$, P2, P3...), le produit final étant Pn.

2. Insistons lourdement sur ce point: les fragments de thèmes que nous retenons chez tel ou tel auteur ne sont pas choisis en raison de leur pertinence scientifique isolée mais en relation avec leur capacité à s'inscrire directement dans le débat d'ensemble.

3. Voir Godelier (1973 : 161-162) qui s'enlise ainsi dans un passage où il est précisément en train de reprocher à des interprètes de Marx de métamorphoser celui-ci en matérialiste vulgaire, en mettant bien sûr dans le même sac ceux qui s'en réclament et ceux qui le contestent.
4. Ceci prolonge l'idée suggérée dans cette revue de considérer les faits techniques comme des « indicateurs sociologiques » (Guille-Escuret 1985).

5. Jamard (1989: 71) a raison de dire que le structuralisme et le marxisme sont des paradigmes « dépassés, bien que par aucun autre », conjointement délaissés au profit d'ambitions subalternes et parcellaires. Mais l'ampleur de la vocation n'a-t-elle pas été la cause de cette double chute, plus que les contenus respectifs? Les hérauts sont fatigués.

6. La France agricole du XIX ${ }^{e}$ siècle est d'ailleurs un cadre que Godelier (1973 : 57) mentionne à ce sujet.

\section{RÉFÉRENCES}

Balibar, É. 1980 Sur les concepts fondamentaux du matérialisme historique. In L. Althusser et É. Balibar (dir.) Lire le Capital, vol. 2. Paris: Maspero (1 ${ }^{\text {re }}$ édition 1968) : 79-226.

Bonte, P. 1973 Problèmes théoriques de la recherche marxiste en anthropologie, La Pensée 171 : 86-104.

- 1975 L'Organisation économique des Touaregs Kel Gress. In R. Cresswell (dir.) Éléments d'ethnologie, tome 1 : Huit terrains. Paris: Armand Colin: 166-215.

- 1999 Travail, techniques et valeur. Contributions (nouvelles) au débat sur rites et techniques. In J.-L. Jamard, A. Montigny et F.-R. Picon (dir.) Dans le sillage des techniques. Hommage à Robert Cresswell. Paris: L'Harmattan: 15-30.

Cresswell, R. 1975 Rapports techniques et rapports sociaux: l'exemple de l'Irlande. In A. Casanova, C. Jest et G. Ravis-Giordani (dir.) Ethnologie et histoire. Forces productives et problèmes de transition. Paris: Éditions Sociales: $521-537$.

- 1996 Prométhée ou Pandore? Propos de technologie culturelle. Paris: Éditions Kimé (« Anthropologie »).

Giraud, M. \& Jamard, J.-L. 1985 Travail et servitude dans l'imaginaire antillais. Une littérature orale en question, L'Homme 96: 77-96.

Godelier, M. 1973 Horizon, trajets marxistes en anthropologie. Paris: Maspero.

Guille-Escuret, G. 1985 La Variabilité des pratiques techniques comme indicateur sociologique dans les Hautes Corbières viticoles (Aude), Techniques \& culture 5: 111-123.

- 1990 Palmier à huile, vin de palme, et transformations sociales en Lobaye (forêt centrafricaine), Informations sur les Sciences Sociales 29 (2): 327-353.

- 1993 Technical innovation and cultural resistance: the social weight of plowing in the vineyards of Les Corbières (Languedoc). In P. Lemonnier (dir.) Technological choices: arbitrariness in technology from neolithic to high-tech. London: Routledge: 214-226.

- 1998 La Révolution agricole des Pygmées aka: de la structure dans l'événement et réciproquement, L'Homme 147: 105-126.

— 1999 Introduction: Anthropologies réfutables et science falsifiée, Diogène 188: 3-19. 
— 2001 Technologie et économie: l'homme producteur. In M. Segalen (dir.) Ethnologie. Concepts et aires culturelles. Paris: Armand Colin: 95-115.

Jamard, J.-L. 1989 Parménide, Héraclite et l'anthropologie française: $2^{e}$ partie, Gradhiva 7 : 39-72.

- 1993 Anthropologies françaises en perspective. Presque-Sciences et autres Histoires. Paris: Éditions Kimé («Anthropologie »).

Meillassoux, C. 1964 Anthropologie économique des Gouro de Côte-d'Ivoire. Paris: Mouton.

Panoff, M. 1985 Du travail villageois au travail en plantation (Mélanésie), Techniques \& culture 5 : 25-137.

Terray, E. 1969 Le Marxisme devant les sociétés primitives. Paris: Maspero.

\section{RÉSUMÉ}

Retour aux modes de production, sans contrôle philosophique. La problématique marxiste a révélé son incontestable supériorité en ethnologie économique avant de s'effondrer pour des motifs fort éloignés de l'activité scientifique. Comme aucune autre approche n'a pu la remplacer de façon convenable, l'ethnologie économique elle-même se retrouve sinistrée. Il est grand temps de revenir à la méthode d'analyse des modes de production, en profitant de ce qu'elle a cessé de supporter des joutes scolastiques, l'objectif étant de chercher comment elle peut être utile à la sociologie comparative, et non plus l'inverse. Entre procès de travail concurrents et dualités des modes de production, l'étage des procès de production a été négligé, bien qu'il conditionne l'interdisciplinarité, notamment avec la technologie culturelle et l'écologie humaine. La complémentarité de deux sources d'illustrations est ici mise à profit: les Corbières viticoles (Languedoc) et la forêt centrafricaine près de la Lobaye.

\section{ABSTRACT}

Back to modes of production, without a philosophical control. The Marxism proved beyond all doubt its superiority for studies in economical anthropology, before it collapsed, for reasons far from science. As no other approach has usefully replaced it, economic anthropology is in ruins. It is time to return to the analysis of the modes of production, since it stopped supporting scholastic jousting. The aim is to find how such analysis can help, not harm, comparative sociology. Between rival processes of labour and dualities of modes of production, the processes of production have been overlooked, although they are the only way toward interdiciplinarity, especially with cultural technology and human ecology. Two complementary examples are presented to illustrate the debate: the wine-growing Corbières area in France and the Centrafrican forest near the Lobaye.

\section{MOTS CLÉS}

Centrafrique, Corbières, France, marxisme, modes de production.

\section{KEYWORDS}

Centrafrica, Corbières area, France, marxism, modes of production.

\section{NOTE(S) ET RÉÉÉRENCES DE LA PRÉSENTATION}

En ouverture : Campement pygmée.

Nos vifs remerciements à Claude-Marcel Hladik pour cette photographie prise en Lobaye (Centrafrique) vers 1983. 